\title{
Effect of Nimodipine on Premature Luteinizing Hormone Surge in Women Undergoing Intrauterine Insemination
}

\author{
Zahra Razghandi $^{1^{\circledR}}$, Robabeh Taheripanah $^{1^{*}}{ }^{\circledR}$ Zahra Heidar $^{\circledR}$
}

\begin{abstract}
Objectives: To determine the effect of nimodipine on premature luteinizing hormone (LH) surge in women undergoing intrauterine insemination (IUI).

Patients and Methods: Fifty-six infertile women participated in this randomized clinical trial after referring to Mahdiyeh hospital, Tehran, Iran and undergoing IUI treatment in 2017. Participants were randomly divided into nimodipine $(\mathrm{n}=34)$ and placebo $(\mathrm{n}=22)$ groups. The demographic and clinical profile of women were collected using a predesigned checklist. In the nimodipine group, 30 mg tablets were given to patients three times daily for 2 days. Finally, the serum levels of LH and estradiol were measured before and after the intervention.

Results: Based on the results, the LH surge was observed in 8 (34.8\%) women in the placebo group $(P=0.04)$ while it was not detected in $29(78.4 \%)$ women in the nimodipine group. There were no statistical differences in the serum levels of estradiol and LH between the 2 groups before the intervention. The serum levels of estradiol in both groups increased after intervention although this increase was not significant. Eventually, no statistical difference was found between the 2 groups in terms of fertility rate.

Conclusions: In general, nimodipine can significantly reduce premature LH surge in patients undergoing IUI compared to the placebo group.

Keywords: Nimodipine, LH surge, Intrauterine insemination, Gonadotropin-releasing hormone agonist (GnRH) agonist, GnRH antagonist, Calcium channel blocker, Iran
\end{abstract}

\section{Introduction}

Childbearing is an important subject with cultural, social, political, and economic dimensions, particularly in traditional societies (1). Infertility is a prevalent condition in reproductive medicine, which can affect the lives of both males and females. The infertility rate ranges from $10 \%$ to $20 \%$ in the reproductive population (2). In addition, infertility is considered as a major lifestyle crisis that might trigger some problems for many individuals resulting in family and social problems in Iran (3-6).

Today, advances in infertility treatment, especially through improving assisted reproductive technology allow many previously infertile couples to have children $(7,8)$, and intrauterine insemination (IUI) has become the first-line treatment option for unexplained infertility $(9,10)$. This technique is also frequently used for the treatment of cervical infertility, moderate male factor infertility, dysovulation, and infertility caused by moderate endometriosis (11). It further involves placing sperm inside a woman's uterus at the time of ovulation in a natural menstrual cycle or following ovulation stimulation. It is also a less invasive option compared to in vitro fertilization (12).

Previous studies indicated that abnormalities in either follicular phase luteinizing hormone (LH) levels or the LH surge have an adverse effect on the development of the oocyte and its fertilizing ability. Some LH surge abnormalities have an important role in unexplained and endometriosis-associated infertility (13).

An important factor in the success rate of IUI technique is to prevent the premature LH surge because premature LH surge can result in the failure of the IUI procedure. In this regard, co-treatment with gonadotropin-releasing hormone agonist (GnRH)-antagonists, as a useful pharmacological approach, can reduce the risk of a premature increase in LH levels (14).

Nimodipine is a lipophilic calcium channel blocker that is used to prevent the spontaneous LH surge and an increase in the premature $\mathrm{LH}$ in women. It inhibits the pulsatile activity of GnRH gene expression and the influx of extracellular calcium and subsequently the stimulation of GnRH release $(15,16)$. A recent publication has demonstrated that nimodipine could be used as a costeffective oral medication which effectively suppresses the release of $\mathrm{GnRH}$ and thus reduces the premature $\mathrm{LH}$ surge in women undergoing IUI. The resulted suggest that the nimodipine may lead to better success following the treatment with IUI and higher rates of a successful

Received 10 September 2019, Accepted 11 January 2020, Available online 29 January 2020

${ }^{1}$ Infertility and Reproductive Health Research Center, Shahid Beheshti University of Medical Sciences, Tehran, Iran.

*Corresponding Author: Robabeh Taheripanah, Tel: +989121096385; Email: taheripanahf@gmail.com 
pregnancy (15).

The present study was conducted to determine the effect of nimodipine in preventing the premature $\mathrm{LH}$ surge among patients undergoing IUI.

\section{Patients and Methods}

Study Design and Population

Infertile women referring to Mahdiyeh hospital, Tehran, Iran, for IUI treatment in 2017, participated in this prospective randomized double-blind clinical trial. The inclusion criteria were patients who were 20 to 40 years old, had both healthy ovaries based on patients' medical history and ultrasound evaluation, had at least one open tube in hysterosalpingography, regular menstrual cycle with a menstrual cycle pattern between 21 and 35 days for at least 3 consecutive months, and were diagnosed with infertility and received the IUI treatment method by the physician.

On the other hand, the exclusion criteria were any follicles greater than $15 \mathrm{~mm}$, body mass index $>38$, polycystic ovarian disease, infertility diagnosis with in vitro fertilization indication, abnormal uterine cavity/ tube pathology, severe infertility due to male factors, and severe underlying disease which disrupt the metabolism of nimodipine such as liver diseases, hypertension, epilepsy, and depression.

The sample size was estimated as 23 patients per group considering a $15 \%$ probability of infertility in a normal community, $65 \%$ fertility probability among our patients after treatment, and a 95\% confidence interval.

To cover patient attrition, 35 patients were assigned to each group, and finally, data from 34 and 22 patients in nimodipine and placebo groups were assessed in this study.

\section{Implementation}

Considering the inclusion and exclusion criteria, eligible participants were randomly divided into the nimodipine and the placebo groups. Then, demographic and clinical information of the patients was collected using a predesigned checklist.

First, patients were assessed using vaginal ultrasonography in order to evaluate the follicles and endometrial thickness. They were excluded from the cycle in the case of the presence of any follicle more than $15 \mathrm{~mm}$ in diameter. Otherwise, their level of $\mathrm{LH}$ and estradiol was measured and human menopausal gonadotropin (75-150 units) was injected based on patients' age and weight from the second day of menstruation for five days. On day 6 of ovulation stimulation, the number and size of follicles were examined using vaginal ultrasonography.

In the presence of at least 2 follicles with a diameter of $14 \mathrm{~mm}$ or higher, patients in the nimodipine group received nimodipine tablet $(30 \mathrm{mg}, 3$ times a day for 2 days), according to the protocol by $\mathrm{ICH}$ GCP(17), and patients in the control group only received the placebo. After day 2, women in the nimodipine group were examined using vaginal ultrasonography. Human menopausal gonadotropin (RONAL-F) and nimodipine tablet were continued until detecting at least two $18 \mathrm{~mm}$ follicles. The vaginal ultrasonography was repeated every 2 days. If at least two $18 \mathrm{~mm}$ follicles were observed, the levels of LH and estradiol were measured using the ELISA test and 2 hCG doses (5000 units each) were injected on the same day, followed by performing IUI after 36 hours. The participants stopped taking their drugs after $\mathrm{LH}$ surge (increased serum LH levels by up to 3 times). The patient's compatibility with drugs and the side effects were recorded using a standard questionnaire.

\section{Statistical Analysis}

The collected data were entered in SPSS, version 21 (Armonk, NY, IBM Corporation). First, the KolmogorovSmirnov test was used for testing the normality of data distribution. Then, the chi-square and $t$ tests were used to compare normally distributed data. Finally, the MannWhitney $U$ test was applied for not normally-distributed data and $P<0.05$ was considered statistically significant.

\section{Results}

The data of 34 and 22 patients in the nimodipine and placebo groups were assessed in the present study. The mean age of the participants was $29.2 \pm 3.1$ and $28.3 \pm 4.5$ years in nimodipine and placebo groups, respectively. The clinical characteristics of the study population according to study groups are presented in Table 1 . Thirty $(81.1 \%)$ and $19(82.6 \%)$ women in nimodipine and the placebo groups had primary and 7 (18.9\%) and 4 (17.4\%) women had secondary infertility, respectively.

As shown in Table 2, the LH surge was not found among $29(78.4 \%)$ women in the nimodipine group while it

Table 1. Clinical Characteristics of the Study population According to Study Group

\begin{tabular}{lccc}
\hline \multirow{2}{*}{ Characteristics } & \multicolumn{2}{c}{ Study Group } & \\
\cline { 2 - 3 } & $\begin{array}{c}\text { Nimodipine } \\
\text { Mean } \pm \text { SD }\end{array}$ & $\begin{array}{c}\text { Placebo } \\
\text { Mean } \pm \text { SD }\end{array}$ & \\
\hline Pregnancy (N) & $0.57 \pm 0.7$ & $0.48 \pm 0.7$ & 0.9 \\
Delivery (N) & $0.14 \pm 0.3$ & $0.26 \pm 0.4$ & 0.5 \\
Abortions (N) & $0.68 \pm 1.6$ & $0.22 \pm 0.5$ & 0.06 \\
Primary infertility (N) & $2.5 \pm 1.1$ & $3.2 \pm 2.3$ & 0.6 \\
Secondary infertility (N) & $2.4 \pm 1.6$ & $1.1 \pm 1.5$ & 0.4 \\
Weight (kg) & $68.4 \pm 9.4$ & $64.8 \pm 8.7$ & 0.5 \\
Follicle (N) & $5 \pm 3.4$ & $4.8 \pm 2.9$ & 0.3 \\
\hline
\end{tabular}

Note. SD: standard deviation

Table 2. The Status of LH Surge According to Study Group

\begin{tabular}{lcccc}
\hline \multirow{2}{*}{ LH Surge } & \multicolumn{2}{c}{ Study Group } & & \\
\cline { 2 - 3 } & Nimodipine & Placebo & Total & \multirow{P}{*}{$\boldsymbol{P}$ Value } \\
& No. (\%) & No. (\%) & & \\
\hline Yes & $8(21.6)$ & $8(34.8)$ & $16(26.7)$ & 0.04 \\
No & $29(78.4)$ & $15(65.2)$ & $44(73.3)$ & \\
\hline Note. LH: luteinizing hormone. & & &
\end{tabular}


Table 3. The Status of the Serum Level of Estradiol and LH Before and After the Intervention According to Study Group

\begin{tabular}{|c|c|c|c|c|}
\hline & & \multicolumn{2}{|c|}{ Study Group } & \multirow[b]{2}{*}{$P$ Value } \\
\hline & & $\begin{array}{l}\text { Nimodipine } \\
\text { Mean (SD) }\end{array}$ & $\begin{array}{c}\text { Placebo } \\
\text { Mean (SD) }\end{array}$ & \\
\hline \multirow{2}{*}{ The serum level of LH } & Before intervention & $5.7(3.7)$ & $5.7(2.1)$ & 0.9 \\
\hline & After intervention & $8.6(6.7)$ & $2.6(2.9)$ & 0.001 \\
\hline \multirow{2}{*}{$\begin{array}{l}\text { The serum level of } \\
\text { estradiol }\end{array}$} & Before intervention & $109.8(140.4)$ & $74.8(26.2)$ & 0.2 \\
\hline & After intervention & 771.5 (640.6) & 934.7 (837.7) & 0.4 \\
\hline
\end{tabular}

Note. SD: standard deviation; LH: luteinizing hormone.

was observed in $8(34.8 \%)$ women in the placebo group $(P=0.04)$.

There was no statistical difference in the serum levels of estradiol and LH between the 2 groups before the intervention $(P=0.2, P=0.9$, respectively). Although the serum levels of estradiol in both groups increased after the intervention, this increase was not significant $(P=0.4)$. On the other hand, there was a significant difference in the serum level of LH between the 2 groups after the intervention $(P=0.001)$, the details of which are provided in Table 3. Conversely, no difference was found between the 2 groups in terms of fertility rate $(P=0.2)$.

\section{Discussion}

Premature LH surge during ovulation stimulation is the most important cause of the failure of IUI treatment. Accordingly, GnRH agonists or GnRH antagonists are currently used to prevent LH surge. Moreover, nimodipine is a calcium channel blocker that can inhibit pulsatile GnRH release in vitro, as well as the LH surge and ovulation in women undergoing IUI $(18,19)$.

Our results showed that there was no significant difference regarding the serum levels of LH between nimodipine and placebo groups before the intervention. In the study by Cantineau and Cohlen, LH surge was found among only one-third of women who were recruited for ovulation induction (20) while LH surge was observed among less than a quarter of women in the nimodipine group. Additionally, Nayot et al reported that taking 60 mg nimodipine 3 times daily for four days prevents $\mathrm{LH}$ surge among women with a regular menstrual cycle (15). The increase in the serum level of LH in the nimodipine group in the above-mentioned study might be due to the dosage of the drug since, in their study, women took a 180 mg daily dosage of nimodipine while, in the present study, participants took a $90 \mathrm{mg}$ daily dosage.

Based on the results of the present study, there was no difference in the serum level of estradiol between the 2 groups of patients before and after the intervention and the increase in the serum level of estradiol was not significant. It seems that the level of estradiol is less likely to increase when GnRH antagonists are used to prevent premature LH surge since the increase in estradiol secretion depends on the ratio of GnRH to LH $(15,21)$.

In addition, our results showed that there was no difference between the 2 groups of patients receiving nimodipine or placebo in terms of fertility rate. Further, Cantineau and Cohlen found no difference in fertility rates among women with and without LH surge (20). In fact, estrogen in the luteal phase has an important role in adjusting the progesterone endometrial receptor and the growth of a secretory endometrium. Follicular evacuation with steroid hormones and the degradation of granulosa cells somewhat reduce estradiol and progesterone in the luteal phase. This phenomenon is more prominent in the case of long-term ovulation stimulation protocol treatment with GnRH antagonists (15). On the other hand, the level of estradiol, especially estradiol to progesterone is very important. Some studies reported a relationship between the serum levels of estradiol in the luteal phase and the rate of implantation (22). It should be noted that the serum level of estradiol and progesterone in the luteal phase was not measured in the present study thus discussing the effect of these 2 hormones on the rate of implantation and pregnancy is impossible. However, the study showed that the fertility rate was not significantly different between the 2 groups.

The results of the present study demonstrated that oral nimodipine could lead to a decrease in premature LH surge during treatment with IUI and consequently could lead to an increase in successful fertility rates. In other words, it can implicate in infertility treatments through assisted reproductive technology and improve their outcomes

\section{Limitations of the Study}

It is notable that the limitation of our study was the lack of medication by the name of nimodipine with the trade name of Nimotop. Therefore, Cerotop was used instead of Nimotop. Further studies are recommended with a larger sample size for the generalizability of the results. In addition, other randomized clinical trials are needed to determine the effect of nimodipine on premature $\mathrm{LH}$ surge in women undergoing in vitro fertilization.

\section{Conclusions}

In general, the results of the present study showed that nimodipine can significantly reduce premature LH surge in patients undergoing IUI compared to placebo, but the fertility rate did not differ between the cases in the control group. 


\section{Conflict of Interests}

Authors declare that they have no conflict of interests.

\section{Ethical Issues}

The study protocol conformed to the ethical guidelines of the Declaration of Helsinki and written consent was obtained from all women entering the study. This study was approved by the Ethics Committee of Shahid Beheshti University of Medical Sciences, Tehran, Iran and registered in Iranian Registry of Clinical Trials (identifier: IRCT20141223020408N6).

\section{Acknowledgments}

The authors of the manuscript would like to thank the staff of Mahdiyeh hospital, Tehran, Iran and all women who volunteered to participate in the present study.

\section{References}

1. Behjati-Ardakani Z, Navabakhsh $M$, Hosseini SH. Sociological study on the transformation of fertility and childbearing concept in Iran. J Reprod Infertil. 2017;18(1):153-61.

2. Direkvand-Moghadam A DA, Khosravi A. Epidemiology of female infertility: A review of literature. Biosci Biotech Res Asia. 2013;10(2):559.

3. Direkvand-Moghadam A, Delpisheh A, DirekvandMoghadam A. Effect of infertility on the quality of life, a cross- sectional study. J Clin Diagn Res. 2014;8(10):OC13OC15. doi:10.7860/JCDR/2014/8481.5063

4. Namavar Jahromi B, Mansouri M, Forouhari S, Poordast T, Salehi A. Quality of life and its influencing factors of couples referred to an infertility center in Shiraz, Iran. Int J Fertil Steril. 2018;11(4):293-7. doi:10.22074/ijfs.2018.5123

5. Amiri M, Khosravi A, Chaman R, et al. Social Consequences of infertility on families in Iran. Glob J Health Sci. 2015;8(5):89-95.

6. Aflakseir A, Zarei M. Association between Coping Strategies and Infertility Stress among a group of women with fertility problem in Shiraz, Iran. J Reprod Infertil. 2013;14(4):202-6.

7. Silva SGD, Bertoldi AD, Silveira MFD, Domingues MR, Evenson KR, Santos ISD. Assisted reproductive technology: prevalence and associated factors in Southern Brazil. Rev Saude Publica. 2019;53:13.

8. Szamatowicz M. Assisted reproductive technology in reproductive medicine - possibilities and limitations. Ginekol Pol. 2016;87(12):820-3. doi: 10.5603/GP.2016.0095

9. Atasever M, Kalem MN, Hatirnaz S, Hatirnaz E, Kalem Z, Kalaylioglu Z. Factors affecting clinical pregnancy rates after IUI for the treatment of unexplained infertility and mild male subfertility. J Turk Ger Gynecol Assoc. 2016;17(3):134-8. doi: 10.5152/jtgga.2016.16056

10. Cohlen B, Bijkerk A, Van der Poel S, Ombelet W. IUI: review and systematic assessment of the evidence that supports global recommendations. Hum Reprod Update.
2018;24(3):300-19. doi: 10.1093/humupd/dmx041

11. Merviel P, Cabry R, Lourdel E, et al. Intrauterine insemination. Rev Prat. 2014;64(1):87-91.

12. Kim D, Child T, Farquhar C. Intrauterine insemination: a UK survey on the adherence to NICE clinical guidelines by fertility clinics. BMJ Open. 2015;5(5):e007588. doi: 10.1136/bmjopen-2015-007588.

13. Yu TN, Liu YL, Wang $\mathrm{PH}$, Chen $\mathrm{CH}$, Chen $\mathrm{CH}$, Tzeng CR. A novel strategy of using corifollitropin alfa in the ultrashort gonadotropin-releasing hormone agonist (GnRHa) protocol in unselected patients: a patient-friendly alternative. Taiwan J Obstet Gynecol. 2019;58(5):656-61. doi: 10.1016/j.tjog.2019.07.013.

14. Depalo R, Trerotoli P, Chincoli A, Vacca MP, Lamanna G, Cicinelli E. Endogenous luteinizing hormone concentration and IVF outcome during ovarian stimulation in fixed versus flexible GnRH antagonist protocols: An RCT. Int J Reprod Biomed (Yazd). 2018;16(3):175-82.

15. Nayot D, Klachook S, Casper RF. Nimodipine, a calcium channel blocker, delays the spontaneous LH surge in women with regular menstrual cycles: a prospective pilot study. Reprod Biol Endocrinol. 2013;11:7. doi: 10.1186/14777827-11-7

16. Fainaru O, Firestone R, Casper RF. Oral nimodipine inhibits the ovarian cycle in mice. Fertil Steril. 2011;95(4):1494-6.

17. Network GCP. Using Nimodipine, a Calcium Channel Blocker, to Prevent the LH Surge and Ovulation in Women Undergoing Assisted Reproduction 2019. https://ichgcp. net/clinical-trials-registry/NCT01551368. Updated Nov 9, 2019.

18. Fukushima A, Sano A, Aiba S, Kimura F. Role of Na+ and $\mathrm{Ca}^{2+}$ channels in the preoptic LH surge generating mechanism in proestrous rats. Endocr J. 2003;50(2):145-53.

19. Haisenleder DJ, Ferris HA, Shupnik MA. The calcium component of gonadotropin-releasing hormone-stimulated luteinizing hormone subunit gene transcription is mediated by calcium/calmodulin-dependent protein kinase type II. Endocrinology. 2003;144(6):2409-16.

20. Cantineau AE, Cohlen BJ. The prevalence and influence of luteinizing hormone surges in stimulated cycles combined with intrauterine insemination during a prospective cohort study. Fertil Steril. 2007;88(1):107-12. doi:10.1016/j. fertnstert.2006.11.136

21. Crawford JL, Heath DA, Haydon LJ, Thomson BP, Eckery DC. Gene expression and secretion of LH and FSH in relation to gene expression of $\mathrm{GnRH}$ receptors in the brushtail possum (Trichosurus vulpecula) demonstrates highly conserved mechanisms. Reproduction. 2009;137(1):129-40.

22. Vanderlelie J, Bell K, Perkins AV. The serum concentration of estradiol after embryo transfer and the decline from preovulatory levels may influence the success of IVF treatment. Horm Res. 2003;59(2):95-9. doi: $10.1159 / 000068578$ creativecommons.org/licenses/by/4.0), which permits unrestricted use, distribution, and reproduction in any medium, provided the original work is properly cited. 